Volume 9, No.1, January - February 2020

International Journal of Advanced Trends in Computer Science and Engineering

Available Online at http://www.warse.org/IJATCSE/static/pdf/file/ijatcse64912020.pdf

https://doi.org/10.30534/ijatcse/2020/64912020

\title{
An Efficient Effort Estimation of a Java Program using Cyclomatic Complexity
}

\author{
P.Veera Sekhar ${ }^{1}$, Vamsidhar Enireddy $^{2}$, K.Bhargav Ram $^{3}$, D.V.SaiSubba Reddy ${ }^{4}$ \\ ${ }^{1}$ Department of Computer Science and Engineering, KoneruLakshmaiah Educational Foundation, Vaddeswaram, \\ Guntur, India, veerasekharpatibandla@gmail.com \\ ${ }^{2}$ Assoc.Professor, Department of Computer Science and Engineering, KoneruLakshmaiah Educational \\ Foundation, Vaddeswaram, Guntur, India, enireddy.vamsidhar@gmail.com \\ ${ }^{3}$ Department of Computer Science and Engineering, KoneruLakshmaiah Educational Foundation, Vaddeswaram, \\ Guntur, India, kbhargavramchowdary@ gmail.com \\ ${ }^{4}$ Department of Computer Science and Engineering, KoneruLakshmaiah Educational Foundation, Vaddeswaram, \\ Guntur, India, dsaireddyd@yahoo.com
}

\begin{abstract}
This present paper proposes to discuss the structured testing methodology for software testing. It is referred to basis path testing. Depend on the cyclomatic complexity measure formal testing utilizes the control flow structure of software set up path exposure criteria. Extension of the primary structured testing technique for integration testing and object-oriented systems are also accessible. A number of connected software complexity metrics are described. We present a survey of software effort estimation and Software size estimation, which is vital characteristics, has been designated for the determination of limiting the examiner standards. In SPM field, management is always needed for the organization knowledge purpose. According to our topic, we had analytically studied two best useful techniques that are software effort estimation and software size estimation
\end{abstract}

Key words : Cyclomatic complexity, Software testing, Software project management, Software effort estimation

\section{INTRODUCTION}

In literature there are number of techniques that are available for the software developers to predict the effort and cost estimation of the project which is an important task for them. These estimation techniques are well-known techniques in order to sort out the precise estimate for determination and progress of essential software organization. Here present software metrics give valuable information about project character and also provide qualified criteria for measuring several software products. Depending on the original estimation, the survey paper is made for a high-quality requirement for equalization of estimates.
In [1] authors came up with a architecture as the structure of parts, and their dependencies, and the standards and aides that control the plan and development in time. As expressed by [2]software design is as a dynamic auxiliary depiction of the product framework as far as its primary segments and the connections among them. Concentrates on quantitative appraisal of programming models are picking up significance because of their job in surveying the nature of design enhancements [3]. IEEE 1471 standard characterizes programming engineering as the crucial association of a framework typified in its segments, their connections to one another and to nature and the standards managing its plan and advancement [4]. From this definition, the segment and the connector are strengthened as the focal ideas of programming design. A segment can be as basic as an article, a class, or a technique, and as intricate as a bundle of classes or procedures. Connectors can be as straightforward as system calls or as detailed as customer server conventions, connects between appropriated databases, or middleware. Programming upkeep is arranged into versatile, remedial, preventive and perfective[5]. Most associations are worried about the expenses of programming upkeep, for it has been expanding consistently and numerous organizations spend roughly $65 \%$ of their product spending plan on support [6]. The procedure of hazard evaluation is valuable in distinguishing complex modules that require point by point investigation, assessing possibly irksome modules. As indicated by the NASA-STD-8719.13A hazard is a component of the foreseen recurrence of event of an undesired occasion, the potential seriousness of coming about results, and the vulnerabilities related with the recurrence and severity. [6].

Accentuation on programming engineering is being put on structure designs, accordingly the conventional act of impromptu programming development is gradually moving towards design situated advancement. The engineering takes into account different free and inexactly coupled segment usage components. Risk assessment evaluation and investigation for programming models is roused by the way that various administrators and sellers may decide to 
convey/create various instruments to accomplish a similar end, and there should be various components to take care of issues at better places in an arranged situation. Moreover, usage bugs or arrangement mistakes might render an execution insufficient. Bigger modules are more change inclined. A bigger module has greater usefulness, in this manner there is more noteworthy probability that some usefulness in the module should be adjusted or improved. Modules taking an interest in configuration designs are less change inclined. Examples are structured so changes are made by means of subclasses or by including new member classes instead of altering effectively present classes. Examples advance simple of progress; henceforth classes partaking in examples ought to require less changes.

\section{WRITING AUDIT}

To address the changing scenario it is important to foresee the maintenance effort cost the Architecture-Level Prediction about programming support (ALPSM) comes into play as the objectives derived from it helps us to build the things discussed above[7]. The principle commitment of this strategy comprise of the design level where this expectation is performed. ALPSM characterizes an upkeep profile, similar to a lot of progress situation errands. A situation portrays an activity, or succession of activities that may happen as identified with the framework. Subsequently a difference in situation depicts a specific upkeep errands. Utilizing the upkeep profile, the engineering is assessed utilizing the situation depicts a specific support exertion for a product framework can be evaluated. The technique has various information sources the prerequisites determinations, the structure of the design, ability from programming engineers and conceivably chronicled support information. This strategy examinations viability by taking a gander at the effect of situations. It utilizes the size of changes as an indicator for the exertion expected to embrace the framework to a situation. The ALPSM doesn't to address chance appraisal [8], thus the need to improve the strategy in order to fuse the hazard evaluation perspective during programming systems for upkeeps.

Technique for Software Maintenance Risk Assessment at the Architecture Level (MSMRAAL) The ALPSM talked about in segment above doesn't give components to address the dangers that are related with the support changes [8].That framework to programming upkeep threat evaluation In the auxiliary building level contains the Emulating steps. 1. Distinguish Classes from the help tasks, beginning with those circumstances. Model the conditions areutilising UML determinations: 2. Coordinate situations: to everything about help assignments, an illustrative set from thecases will make portrayed. 3. Guide those circumstances under the auxiliary structure: for each circumstance decides those portions that would Also impact whatever degree they will an opportunity to be changed, this realizes the range of the impact of the affirmation of the circumstance. 4. Guide those taking interest classes of the cases Similarly as acquainted with UML conclusions model(s) should An appropriated setup plan that best matches those model. 5. Threat appraisal: make that those impact of a change situation; assess the risk on the swell effects of the movements (upkeep) to an opportunity to be chosen for A section for gratefulness with the coordinating portions in order to anticipate those general peril that could be sticked ought to Throughout those help of a structure.

\section{IMPLEMENTATION}

Cyclomatic Complexity nature, sees project capriciousness related to the quantity of control courses through an undertaking module, deduced an item intricacy measure beginning with outline standard using that meaning of the cyclomatic number which compares of the sum about directly self-sufficient approaches to a task. It might be normal on be free for lingo structure. This measure gives a flat out sum that can make diverged from that capriciousness for different tasks. 's cyclomatic intricacy might be a ramifications of a framework module's control-stream multifaceted nature Also need being seen to an opportunity as a trustworthy pointer for flightiness Previously, enormous item undertakings .Recognizing the number about control courses through the program, A 10-line venture for 10 task declarations might be less requesting with seeing all the over a 10-line framework with 10 on the off chance that declarations. MCC might be portrayed for each module with an opportunity to be $\mathrm{M}=\mathrm{E}-\mathrm{n}$ $+\mathrm{X}$, the spot $\mathrm{m}$ is those Cyclomatic multifaceted nature (MCC) metric, e might be the quantity of edges, $n$ will be the number from the nodes or decision centers (restrictive explanations), and $\mathrm{X}$ will be those number of ways out (return articulations) in the outline of the limit exclusively. Control stream diagrams (CFC) portray that method of reasoning structure for item modules. The hubs address computational announcements or articulations, and the edges address trade about control between hubs. Each useful execution method for a programming module needs an contrasting way beginning and those section of the retreat hub of the module's control stream diagram. The focal points of the CFC metric is that it could be used Likewise An upkeep and individual fulfillment metric, it accommodates the overall unpredictability of transform design.

The utilisation about designs toward structural level favours those decrease from the repetitive dependability displaying the worth of effort and the comprehend capability of the unwavering quality model And permits the creator with the motivation behind around deficiency tolerance.Thisallows foreseeing the impacts of the specific structural choices with respect to those unwavering quality of the framework.

A programming model to those test might have been planned done such an approach to empowering the client to enter a. Java document. Those programming model will be executed In the nearby subnet level utilising two diverse instruments Outline design and the secluded methodologies. 


\section{METHODOLOGY}

Provided for those expanding cost for software development, McCabe viewed as that a 'arithmetical method that will give acceptable solution. An quantitative premise to modularization will recognize programming modules that will get a chance to be troublesome to test alternately maintain' might have been obliged. The utilization of A-lines of code (LOC) metric might have been dismissed since McCabe Might perceive no clear relationship in the middle of period Also module multifaceted nature. As an alternative, he proposed that there are a numerical ways to control all the way through a module might make a better indicator, especially as this showed up with make determinedly identified with testing exertion. And, a great deal of the worth of effort around 'structured programming' in the initial 1970s concentrated ahead system control stream structures. Unfortunately, that number for ways through at whatever program with An retrograde limb will be possibly limitless. Fortunately, that issue could make determined by the provision about chart hypothesis. The control stream from claiming whatever procedural bit for a program can be delineated Concerning illustration An guided graph, by speaking to every executable articulation (or aggregation of proclamations the place the stream for control may be sequential) Similarly as a node, and the flow from claiming authority Concerning illustration those edges the middle of them. That cyclomatic complex nature of a chart will be suitable because giving those chart is determinedly connected, and it demonstrates the amount from claiming essential ways (i. E. Linearly free circuits) held inside a graph, which, the point when utilised within combination, might produce the more significant part-time permits ways through those charts alternately project.

That cyclomatic complexity $\mathrm{v}$ of a project chart $\mathrm{g}$ may be.

$\mathrm{V}(\mathrm{G})$ is given as e- $\mathrm{n}+1$

Here e will be those number for edges, and $n$ will be those number of nodes. A determinedly associated chart is particularly case for which provided for At whatever two nodes $r$ and $s$ the present paths from $r$ and $s$ to $r$. Fig. 1 indicates a sample inference about cyclomatic intricacy starting with a basic project.Also its related control chart. Note that that system chart is settled on determinedly associated by were as for an edge interfacing those wind node of the start node. That methodology for including an extra edge of the system chart could be bypassed by adding you quit offering on that one of the cyclomatic intricacies figuring. The count might make summed up to system graphs that hold numerous you leave offering on that one alternately more component, subject of the confinement that every part contain only one and a node for its exit. To a graph Swith a set of connected components, the cyclomatic complexity will be.

$\mathrm{V}(\mathrm{S})$ is given as e-nt ${ }^{2} \mathrm{C}$

Here $\mathrm{C}$ denotes the components that are connected to each other and to represent a program graph containing the multi components e is used here, also it denotes the containment of the subroutines and these are shown in the figure. McCabe observers the reduction of calculation to a simple count for conditions plus one which is argued as a pure compound for example

IF $x, 1$ and Y might have been An daintily guised nested IF, afterwards every state if help module complexity, as opposed just numbering predicates. Similarly, an instance proclamation may be seen as a various though proclamation (i.e., It contributes $\mathrm{n}$ - I will $\mathrm{v}(\mathrm{S})$, the place $\mathrm{n}$ may be the number from claiming cases). [10] An useful requisition of the metric in utilizing it to gatherings give an upper point of confinement will module complexity, Past which a module ought further bolstering a chance to be subdivided under simpler components, an esteem of $\mathrm{v}(\mathrm{S}) 5,10 \mathrm{might}$ have been suggested, Despite he acknowledged that over sure situations, notably massive situation structures, the breaking point could make loose.

Theoretical considerations and those numbering decide for diverse control proclamations bring been that subject from claiming exactly discussion. Myershas contended that An unpredictability interim will be An additional viable measure about multifaceted nature over a straightforward cyclomatic number. Those interim need a more comfortable certain for choice proclamation check also called as Predicate count plus particular case Also an upper bound of singular condition number Also specificsituation. Myers utilized the taking after three illustrations on backing as much modified form of the cyclomatic complexity metric

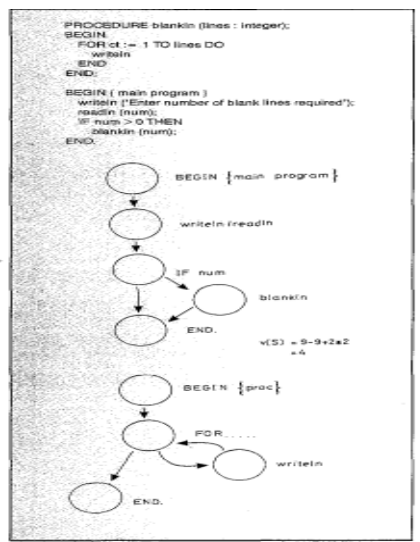

Figure 1: System Control Stream Structures

$$
\begin{gathered}
\text { If } p=0 \text { then...... } \\
\text { Else.........; } \\
V(S)=2 \\
\text { Myers }=(2: 2) \\
\text { If } p=0 \text { and } q>1 \text { then..... } \\
\text { Else.....; } \\
V(S)=3 \\
\text { Myer } s=(2: 3)
\end{gathered}
$$




$$
\begin{gathered}
\text { If } p=0 \text { then...... } \\
\text { If } q>1 \text { then...... } \\
\text { Else..... } \\
\text { Else.....; } \\
V(S)=3 \\
\text { Myers }=(3: 3)
\end{gathered}
$$

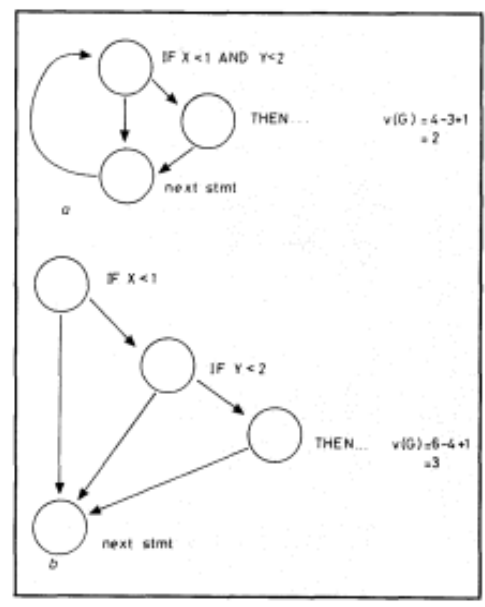

Figure 2: Numbering Predicates

As much contention may be that it is naturally clear that the third illustration may be a greater amount perplexing over those second, a qualification not committed by that cyclomatic amount. The perfect underlying as much An dealt with Similarly as single choice $b$ dealt with as separate choices adjustment seems with a chance to be that there will be an additional possibility to inserting extra else clauses under a system for A larger amount of whether proclamations. They need aid not counted Toward the McCabe metric.Likewise will be showed by the taking after two system fragments, both of which have cyclomatic complexities from claiming 2: compound state dealt with Similarly as a single choice Also Similarly as if $\mathrm{X}<1$ after that else. $(\mathrm{v}(\mathrm{G})=2 \mathrm{v}(\mathrm{G})=2$ Since Myers' intricacy interim doesn't straightforwardly check else proclamations it may be doubtful if it speaks to substantially of a change In that for McCabe's metric. However, those feedback about cyclomatic unpredictability remains, in that it falls flat will recognize the middle of selections with without else limbs. Starting with the point of view by cyclomatic complexity, this will be significant; however, since the number of essential ways stays unalterably testing trouble might not expand. Subsequently, that disappointment about cyclomatic unpredictability with number else limbs is best .On the metric will be exceptional on catch intricacy of appreciation. Need to be proposed that since they were less demanding to see all the over the equal nested IFS, they if help person of the module unpredictability. Considerably of the challenge stems starting with that truth that McCabe might have been initially speculation As far as
Fortran, while most of these challenges emerge from different languages, exactly from claiming them a greater amount recent, for example, such that ADA. T here particular case need should fight for issues for example, such that recognizing the middle of ' $\mathrm{IF} y=1$ or $\mathrm{y}=3$ ' And 'IF $\mathrm{y}=0$ or disaster will be imminent $x / y>1$ '. Those mapping from code will a project chart are vague. Another range about debate may be that $\mathrm{v}=1$ will stay valid to a straight arrangement about at whatever period. Since those metric is uncaring should multifaceted nature contributed from straight successions of statements, a few specialists have suggested adjustments of the straightforward utilization of cyclomatic intricacy. Hansen need suggesting a 2-tuple about cyclomatic multifaceted nature Also operand check (defined to make arithmetical operators, work and subroutine calls, assignments, information and yield proclamations and exhibit subscription). Unfortunately, as dough puncher Also Zweben purpose out, this methodology does fair starting with that issue for 'comparing apples and oranges'. It will be not reasonable how should rank in place about intricacy the 2-tuples (iJ) Furthermore $(1, k)$ the place i $>1$ and $k>j$. Stutter prescribes an elective approach should this specific issue in the type of a cyclomatic stream unpredictability metric. Stream about information may be recognized and to stream of control. Unpredictability for the most part, expansion with an expand long of a straight arrangement of proclamations since a greater amount of information references will about invariably make committed.A further protest of the cyclomatic intricacy metric maybe its self-destructive considerations and conduct towards the structuring about the product. An amount from claiming scientists argue that those cyclomatic multifaceted nature could expansion when applying by acknowledged strategies will enhance project structure. The metric may be uncaring of the utilisation of unstructured strategies, for example, such that bouncing over and out of the circle. Advancement of the unstructured contention will be those protest that the metric overlooks the setting alternately nature's domain of choice. Every last bit choices bring a normal weight, in any case about profundity for nesting alternately association for other choices. Those intricacies of choice can't be recognised to isolation, yet all the must consider different choices inside its growth. This need brought about variants from claiming cyclomatic unpredictability which considers nesting profundity. Hence, a change must make aggravated of the numbering decides. Challenge for testing is an additional perspective from claiming product unpredictability Also particular case with which McCabe might have been fundamentally worried. These different interpretations from claiming cyclomatic multifaceted nature need huge meanings upon the acceptance and requisition of the metric.

As per CC metric then we will form one control flow graph: 


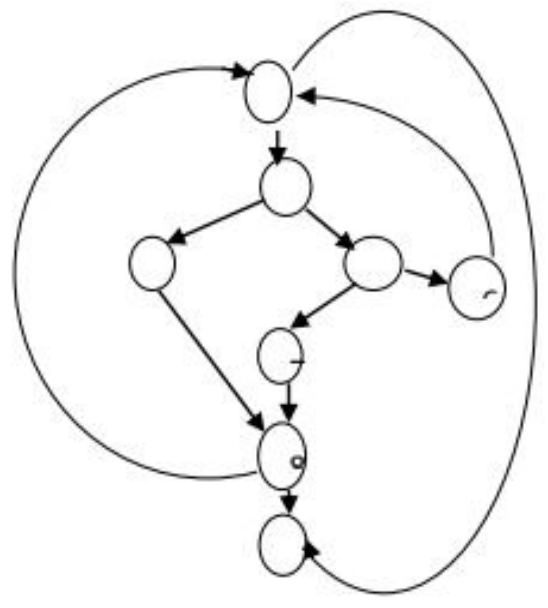

Figure 3: Control Flow Graph

\section{OUTPUT}

\subsection{Interface Requesting For Input:}

6. import java.io.FileInputStream;

7. import java.io.IOException;

8. import java.util.Scanner;

9. import javax.swing.JOptionPane;

10. import org.apache.commons.cli.*;

11. public class TRGeneration \{

12.

13. private static Graph graph;

14. private static TestRequirements tr;

15.

16. public static void main(String[] args) \{

17.

18. //ex.Test1();

19. //ex.Test2();

20. //ex.Test3();

21. //ex.Test4();

22. //ex.Test5(); // Need to work on

23.

24. $\operatorname{tr}=$ new TestRequirements();

25. $\quad$ graph $=$ new $\operatorname{Graph}()$;

26.

27. $/ *$ if (args.length $<1)\{$

28. System.err.println("You must supply an input file");

29. $\quad$ System.exit(1);

30. $\}^{* 1}$

31.

32. Options options = new Options();

33. options.addOption("d", false, "Print debug output"); // does not have a value

34. options.addOption("o", true, "PNG output path"); // does not have a value 35.

36. CommandLineParser parser = new BasicParser();
37. CommandLine $\mathrm{cmd}=$ null;

38. $\operatorname{try}\{$

39. $\quad \mathrm{cmd}=$ parser.parse(options, args);

40. \} catch (ParseException e) \{

41. System.err.println("Caught ParseException: " + e.getMessage () )

42.

43.

44.

45.

46.

47. if (cmd.hasOption("d")) graph.setDebug(true);

48.

49.

50 .

51.

52.

53.

54.

55.

56.

57.

58.

59.

60.

61.

62.

63.

64.

65.

66.

\section{CyclomaticComplexity();}

67. cc.showCyclomaticComplexity $(\operatorname{cc} \cdot \operatorname{check}())$;

68. \}

69.

70.

71.

72.

73.

74.

75.

76.

77.

78.

79.

80.

81.

82.

83. Scanner $\mathrm{s}=$ new Scanner(fstream);

84. while (s.hasNextLine ()$)\{$

85.

86.

87.

88.

89.

90. private static void readSource(String path) \{ System.out.println(path);

FileInputStream fstream = null;

try\{ \} fstream = new FileInputStream ("D://"+path); catch (IOException e) \{ "+path+".ln"+e.getMessage());

System.exit(1);

\} \}

s.close();

try\{

fstream.close();

\}
System.err.println("Unable opening file graph.AddSrcLine(s.nextLine()); 
P.Veera Sekhar et al., International Journal of Advanced Trends in Computer Science and Engineering, 9(1), January - February 2020, 469 - 475

91. catch (IOException e) \{

92. System.err.println("Error closing file "+path+"./n"+e.getMessage());

93. \}

94.

95. \}

\subsection{Interface Requesting For Java File As Input:}

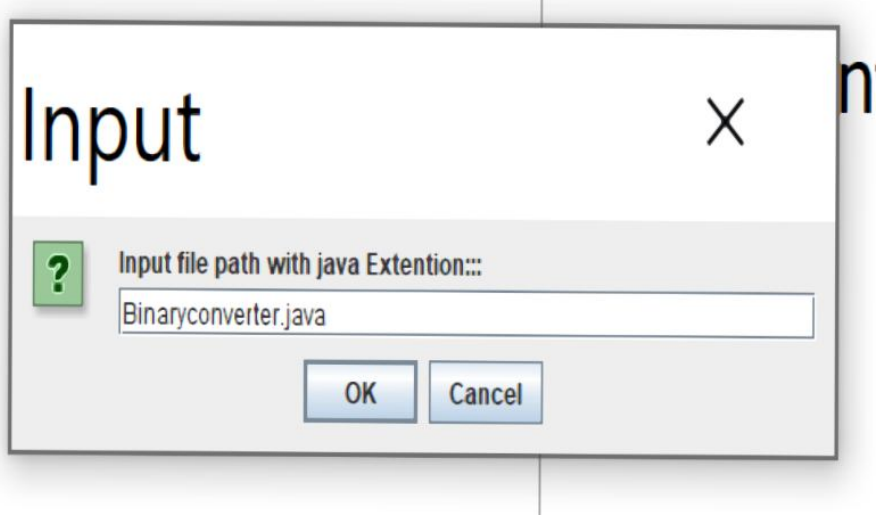

Figure 4: Interface for reading data

\subsection{CFG Graph}

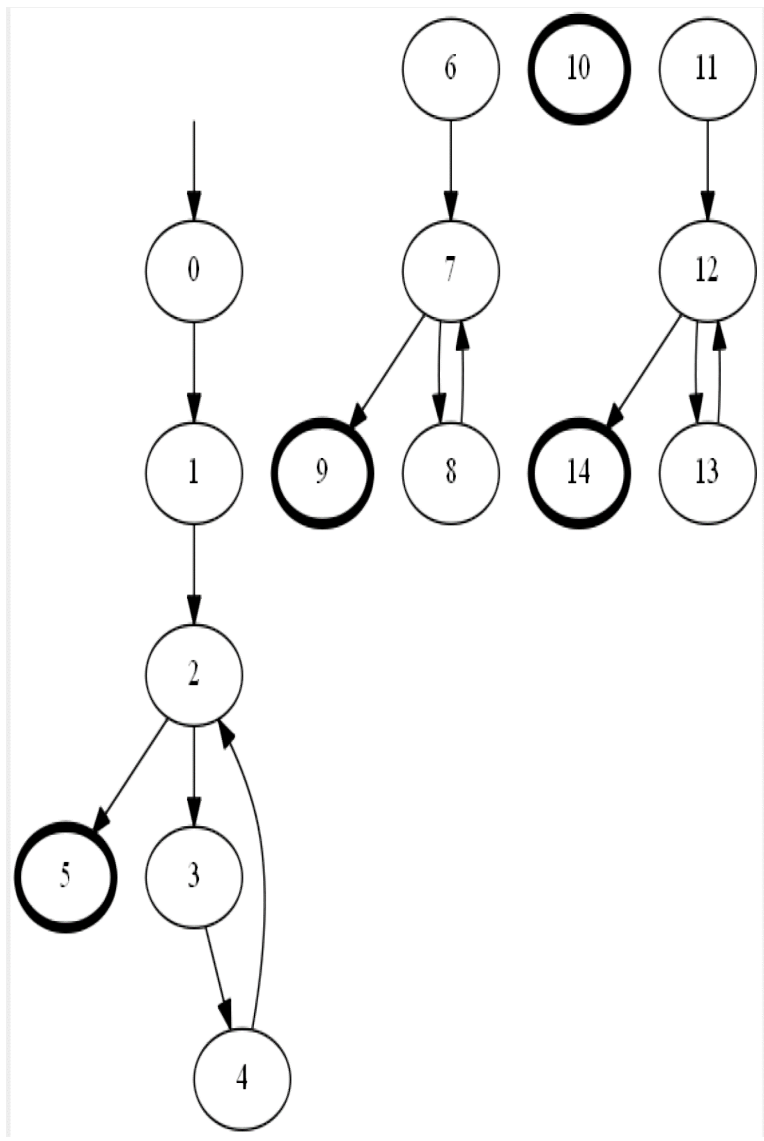

Figure 5: Control Flow Graph of the Input
5.4 Output Displaying The Nodes,Edges By Using The CFG Graph:

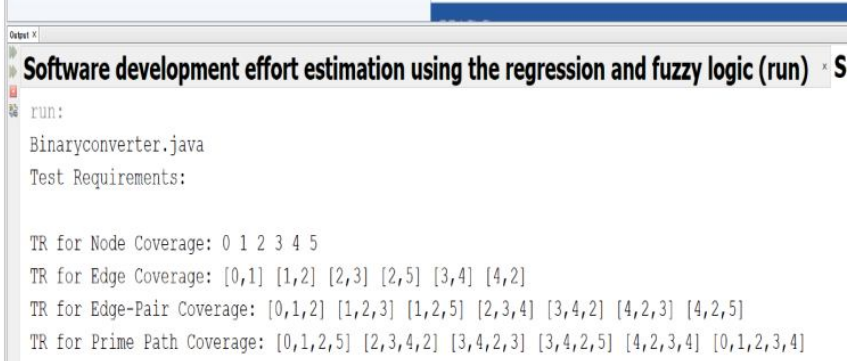

Figure 6 : Showing the Node and Edge using the CFG graph

5.5 Interface Requesting For The Same File As Input For Calculating Complexity:

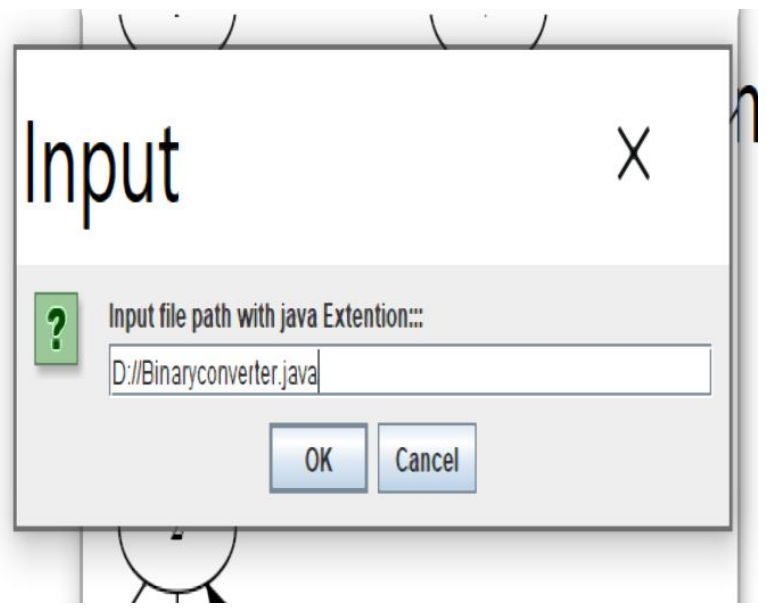

Figure 7: Interface for calculating complexity

\subsection{Interface Displays The Cyclometric Complexity:}

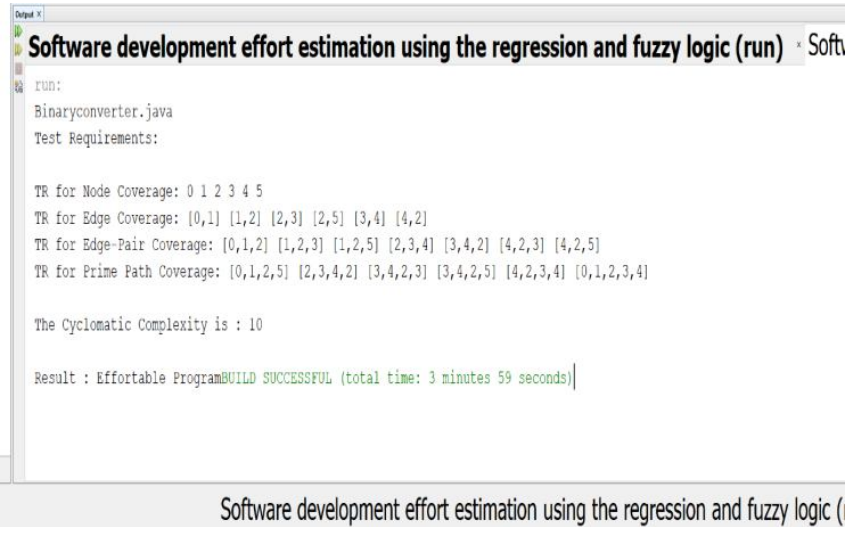

Figure 8: Showing Cyclometric Complexity 


\section{CONCLUSION}

The methodology proposed on this exploration for maintenance effort evaluation assessment on the architectural stage should be confirmed further on distinctive software designs to choose its convenience. Future examinations ought to prescribe the measurements that ought to be remembered for this technique to improve their simplicity of pertinence.

\section{REFERENCES}

1. Garlan, D. Software architecture.In Proceedings of the Conference on the Future of Software Engineering ( 2000)(pp. 91-101). ACM. https://doi.org/10.1145/336512.336537

2. Bass, L., Clements, P., \&Kazman, R. Software architecture in practice. Addison-Wesley Professional (2003).

3. Sant'Anna, C., Figueiredo, E., Garcia, A., \&Lucena, C. . On the Modularity Assessment of Software Architectures: Do my architectural concerns count. In Proc. International Workshop on Aspects in Architecture Descriptions (2007) (AARCH. 07), AOSD (Vol. 7).

4. Emery, D., \& Hilliard, R. Updating architecture frameworks and other topics. In Software Architecture, WICSA . Seventh Working IEEE/IFIP Conference on (2008) (pp. 303-306). IEEE. https://doi.org/10.1109/WICSA.2008.32

5. Sommerville, I. Construction by configuration; Challenges for software engineering research and practice.In Software Engineering . ASWEC. 19th Australian Conference on (2008) (pp. 3-12). IEEE. https://doi.org/10.1109/ASWEC.2008.4483184

6. Abdelmoez, W. M., Goseva-Popstojanova, K., \& Ammar, H. H. (Methodology for maintainability-based risk assessment. In Reliability and Maintainability Symposium, RAMS'06. Annual (2006) (pp. 337-342). IEEE.

7. Bengtsson, P., \& Bosch, J.Architecture level prediction of software maintenance. In Software Maintenance and Reengineering. Proceedings of the Third European Conference on (1999) (pp. 139- 147). IEEE.

8. R. Tombe, S. Kimani and G. Okeyo. Method for Software Maintenance Risk Assessment at the Architecture Level. JIM Journal. Ronald Tombe et al., International Journal of Computer Science and Mobile Computing, Vol.3 Issue.11, pg. 89-101 (C) (2014), IJCSMC All Rights Reserved.

9. Ward W., Software defect prevention using McCabe's complexity metric. Hewlett Packard Journal, 40(2): (1989) 64-69.

10. McCabe T. J. and Bulter C.W. A complexity measure. IEEE Transactions on Software Engineering, 2(4): (1976) 308-320 . https://doi.org/10.1109/TSE.1976.233837 